\title{
Structures in low-energy classical bremsstrahlung
}

\author{
A Florescu†, O I Obolensky $\ddagger$ ब and R H Pratt \\ $\dagger$ Institute for Space Science, Bucharest, Romania \\ $\ddagger$ Department of Physics and Astronomy, University of Pittsburgh, Pittsburgh, \\ PA 15260, USA \\ व A F Ioffe Physical-Technical Institute, St. Petersburg 194021, Russia \\ E-mail: rpratt@pitt.edu
}

Submitted to: J. Phys. B: At. Mol. Opt. Phys.

\begin{abstract}
We study classical bremsstrahlung from neutral atoms and investigate the origins of the structures in the energy dependence of the differential cross section $\omega d \sigma / d \omega$ and the asymmetry parameter $a_{2}$, using soft-photon limit results in terms of elastic scattering. We show that trapping features in elastic scattering do not contribute to the structures. Rather, these structures result from the suppression of contributions to the radiation from certain angular momenta at certain energies. This corresponds to the association of structures in quantum bremsstrahlung with zeroes in certain angular momentum matrix elements at certain energies.
\end{abstract}

\section{Introduction}

Oscillations as a function of incident electron energy $E$ in the differential cross section and the asymmetry parameter are characteristic of the low incident electron energy region (for example, up to $150 \mathrm{eV}$ for $\mathrm{Al}$ ) of the bremsstrahlung from neutral atoms. These structures were first noted in the asymmetry parameter $a_{2}$ [1], considered as a function of $E$ for various fixed ratios of photon energy $\hbar \omega / E$, and they were also identified in the differential cross section $\omega \mathrm{d} \sigma / \mathrm{d} \omega[2]$. Both results were obtained using a classical description of the bremsstrahlung process $[3,4]$. Similar structures have also been observed using a quantum description $[5,6]$. The quantum structures are located at approximately the same energies as the classical ones [6].

A remarkable fact is that both the quantum and classical oscillatory behaviour is largely independent of the ratio $\hbar \omega / E$ and is well characterized by the behaviour in the soft-photon limit $\hbar \omega / E \rightarrow 0$. In the classical case this can be understood as due to the fact that the radiation is mainly emitted in the vicinity of the turning point of electron motion (when its acceleration is greatest), where the electron kinetic energy is much larger than the initial electron energy, and hence it is much larger than all physically allowed energies of radiation [7]. Thus explaining the soft-photon limit behaviour should provide some general understanding of low-energy bremsstrahlung.

In previous work [6] we showed that the quantum structures are related to the zeroes of the radial matrix elements which occur for particular angular momenta at particular energies. That is, in the quantum case the lack of contribution from some 
of the dominant radiative transitions (due to zeroes in corresponding matrix elements) results in the observed structures in the energy dependence of the bremsstrahlung cross section and asymmetry parameter. In this paper, by examining the soft-photon limit of the classical bremsstrahlung, we establish a corresponding origin for the classical structures. We show that the classical structures have, essentially, the same origin as the quantum ones, namely, the lack of contribution from a certain range of angular momentum. In the soft-photon limit we can relate these structures to the behaviour of the scattering angle in certain ranges of angular momentum as incident energy changes.

\section{Brief review of the classical formalism and the soft-photon limit}

A complete description of the classical bremsstrahlung formalism and the softphoton limit of dipole radiation is given in [4]. An extensive analysis of classical bremsstrahlung in screened atomic potentials, under the assumption of energy loss independent trajectories, can be found in $[1,2]$. Here we simply recall these results and give the soft-photon limit of the bremsstrahlung cross section.

The doubly differential cross section describing the radiation emitted into a solid angle $d \Omega_{k}$, in the frequency interval $(\omega, \omega+d \omega)$, is

$$
\omega \frac{\mathrm{d}^{2} \sigma}{\mathrm{d} \Omega_{k} \mathrm{~d} \omega}=\frac{1}{4 \pi} \omega \frac{\mathrm{d} \sigma}{\mathrm{d} \omega}\left[1+\frac{a_{2}}{2} P_{2}\left(\cos \theta_{k}\right)\right] .
$$

Here $P_{2}$ is the Legendre polynomial and $\theta_{k}$ is the azimuthal emission angle of the photon. The bremsstrahlung spectrum

$$
\omega \frac{\mathrm{d} \sigma}{\mathrm{d} \omega}=\frac{8 \hbar \pi e^{2}}{3 m^{2} c^{3}} \mathcal{I}_{1}
$$

where $e$ is the electron charge, $m$ is the electron mass, $v$ is the incident electron velocity at infinity, and $c$ is the velocity of light.

$$
\mathcal{I}_{1}=\frac{2 \pi}{\hbar^{2} v^{2}} \int_{0}^{\infty}\left|\overrightarrow{a_{\omega}}\right|^{2} L \mathrm{~d} L,
$$

where the electron angular momentum $L=m v \rho$ ( $\rho$ is the classical impact parameter), and

$$
\vec{a}_{\omega}=\frac{1}{2 \pi} \int_{-\infty}^{\infty} \vec{a}(t) e^{i \omega t} \mathrm{~d} t
$$

is the Fourier transform of the electron acceleration.

The asymmetry parameter $a_{2}$, which characterizes the angular distribution of radiation, is given as

$$
a_{2}=\frac{\mathcal{I}_{2}}{\mathcal{I}_{1}},
$$

where

$$
\mathcal{I}_{2}=\frac{2 \pi}{\hbar^{2} v^{2}} \int_{0}^{\infty}\left[\left|\vec{a}_{\omega}\right|^{2}-3 a_{\omega z}^{2}\right] L \mathrm{~d} L
$$


and $a_{\omega z}$ is the $z$-component of the vector $\vec{a}_{\omega}$, i.e. the component along the incident electron direction.

In the soft-photon limit (s-ph) $\omega \rightarrow 0$ (4) gives us

$$
\vec{a}_{\omega}^{\mathrm{s}-\mathrm{ph}}=\frac{1}{2 \pi} \int_{-\infty}^{\infty} \frac{\mathrm{d} \vec{v}}{\mathrm{~d} t} \mathrm{~d} t=\frac{1}{2 \pi}\left(\vec{v}_{f}-\vec{v}_{i}\right),
$$

where $\vec{v}_{i}$ and $\vec{v}_{f}$ are respectively the initial and final electron velocities. Neglecting energy loss on the trajectories, so that $\left|\vec{v}_{i}\right|=\left|\vec{v}_{f}\right|=v$, one obtains

$$
\left|\vec{a}_{\omega}^{\mathrm{s}-\mathrm{ph}}\right|^{2}=\frac{1}{2 \pi^{2}} v^{2}[1-\cos \Phi(E, L)],
$$

where for angular momentum $L$ the classical scattering angle $\Phi(E, L)$ [1], obtained by

elimination of the time-dependence in the classical equations for motion in a central field $V_{\text {eff }}(r)$, is

$$
\Phi(E, L)=\sqrt{\frac{2}{m}} L \int_{r_{0}(E, L)}^{\infty} \frac{\mathrm{d} r}{r^{2} \sqrt{E-V_{\mathrm{eff}}(r, L)}}-\pi .
$$

Here

$$
V_{\text {eff }}(r, L)=V(r)+\frac{L^{2}}{2 m r^{2}},
$$

$V(r)$ is the atomic potential, and $r_{0}(E, L)$ is the classical turning point defined by

$$
E=V_{\text {eff }}\left(r_{0}(E, L), L\right) .
$$

Thus, in the soft-photon limit, (3) and (6) become [2]

$$
\begin{aligned}
& \mathcal{I}_{1}^{\mathrm{s}-\mathrm{ph}}(E)=\frac{1}{\pi \hbar^{2}} \int_{0}^{\infty}[1-\cos \Phi(E, L)] L \mathrm{~d} L \\
& \mathcal{I}_{2}^{\mathrm{s}-\mathrm{ph}}(E)=\frac{1}{2 \pi \hbar^{2}} \int_{0}^{\infty}[1-\cos \Phi(E, L)][3 \cos \Phi(E, L)-1] L \mathrm{~d} L .
\end{aligned}
$$

The spectrum and the asymmetry parameter in the soft-photon limit are obtained by replacing $\mathcal{I}_{1}$ and $\mathcal{I}_{2}$ in (2) and (5) with $\mathcal{I}_{1}^{\mathrm{s}-\mathrm{ph}}$ and $\mathcal{I}_{2}^{\mathrm{s}-\mathrm{ph}}$ respectively. Hence they are simply given in terms of quadrature integrals over the scattering angle [2] as a function of angular momentum.

\section{The origin of the structures in classical bremsstrahlung}

This section is devoted to an examination of the structures in the spectrum and asymmetry parameter, utilizing their soft-photon limit. We have used (12) and (13) to obtain further results for a number of neutral atoms in order to elucidate the origin of the features we want to explore. The results for $\mathrm{Al}(\mathrm{Z}=13)$ are shown in figures 1 and 2. These results are consistent with the previous classical results $[1,2,6]$, but they are calculated for a more extended energy range (in $[1,2]$ results were obtained only for $E>E_{\mathrm{M}}$, the maximum trapping energy, as defined below). Both the asymmetry parameter and the cross section show a non-monotonical energy dependence (a rise to 


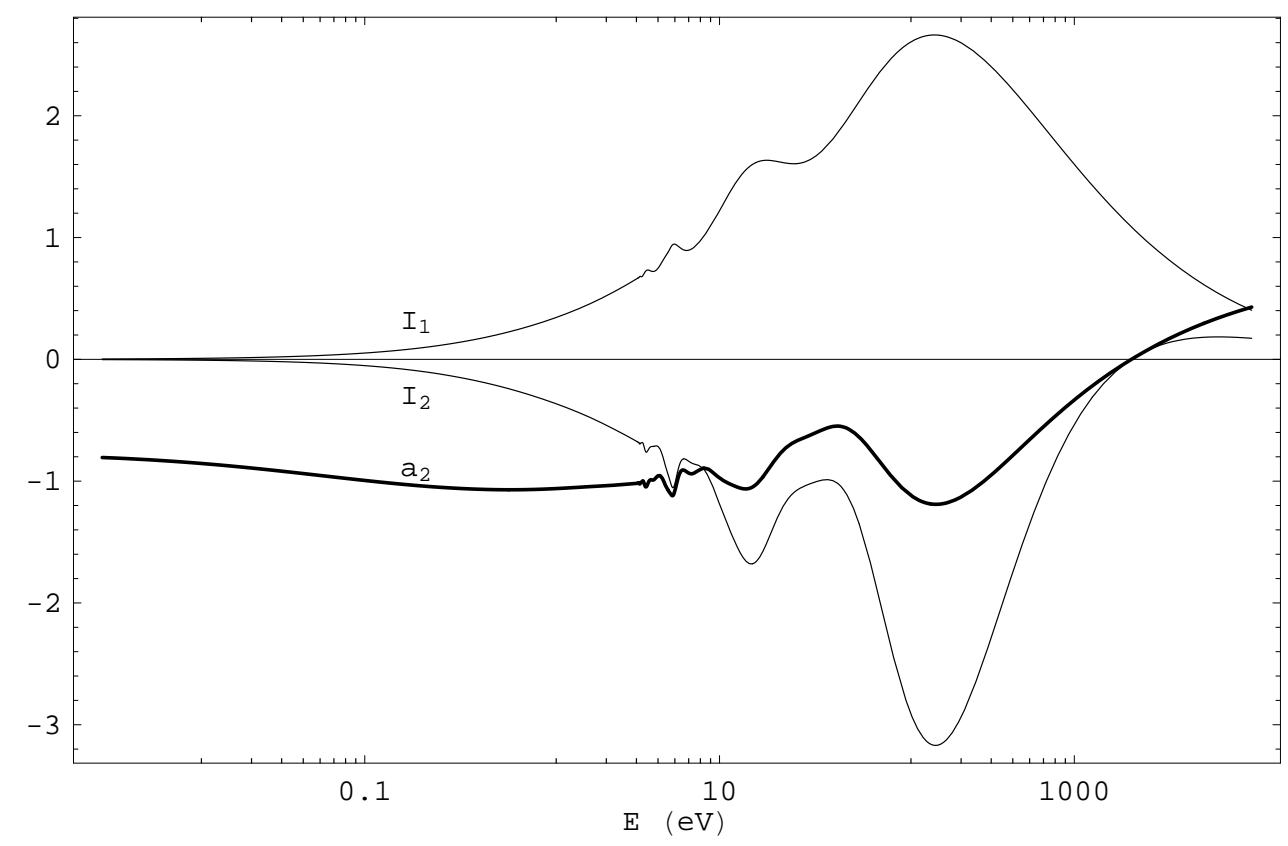

Figure 1. Energy dependence of $\mathcal{I}_{1}^{\mathrm{s}-\mathrm{ph}}$ and $\mathcal{I}_{2}^{\mathrm{s}-\mathrm{ph}}$ (thin lines) and the resulting asymmetry parameter (thick line) $a_{2}^{\mathrm{s}-\mathrm{ph}}=\mathcal{I}_{2}^{\mathrm{s}-\mathrm{ph}} / \mathcal{I}_{1}^{\mathrm{s}-\mathrm{ph}}$, for $\mathrm{Al}$ in a HartreeFock potential.

a peak modulated by a series of local minima in the case of the cross section) up to $E \sim 150 \mathrm{eV}$, as had been seen for other $\hbar \omega / E$.

In the soft-photon case the scattering angle $\Phi(E, L)$ is the basic building block for all other physical quantities. Thus we shall first discuss the behaviour of $\Phi(E, L)$ in a screened neutral potential, using the point Coulomb case results (see [8]) as a reference. Then we shall discuss the resulting features in the integrands (12) and (13), identifying which features are responsible for the observed structures in the cross section and asymmetry parameter.

\subsection{The scattering angle}

In the Coulombic case $V(r)=-Z e^{2} / r$, the turning point

$$
r_{0}^{\operatorname{Coul}}(E, L)=\zeta\left(\sqrt{1+\lambda^{2} L^{2}}-1\right)
$$

and the scattering angle (9)

$$
\Phi^{\operatorname{Coul}}(E, L)=2 \arcsin \frac{1}{\sqrt{1+\lambda^{2} L^{2}}}
$$

where

$$
\lambda^{2}=\frac{2 E}{m Z^{2} e^{4}}, \quad \zeta=\frac{Z e^{2}}{2 E} .
$$

In a screened potential $\Phi(E, L)$ can not be found analytically, except for some limiting cases discussed below. Unlike the Coulomb case, where the scattering angle 


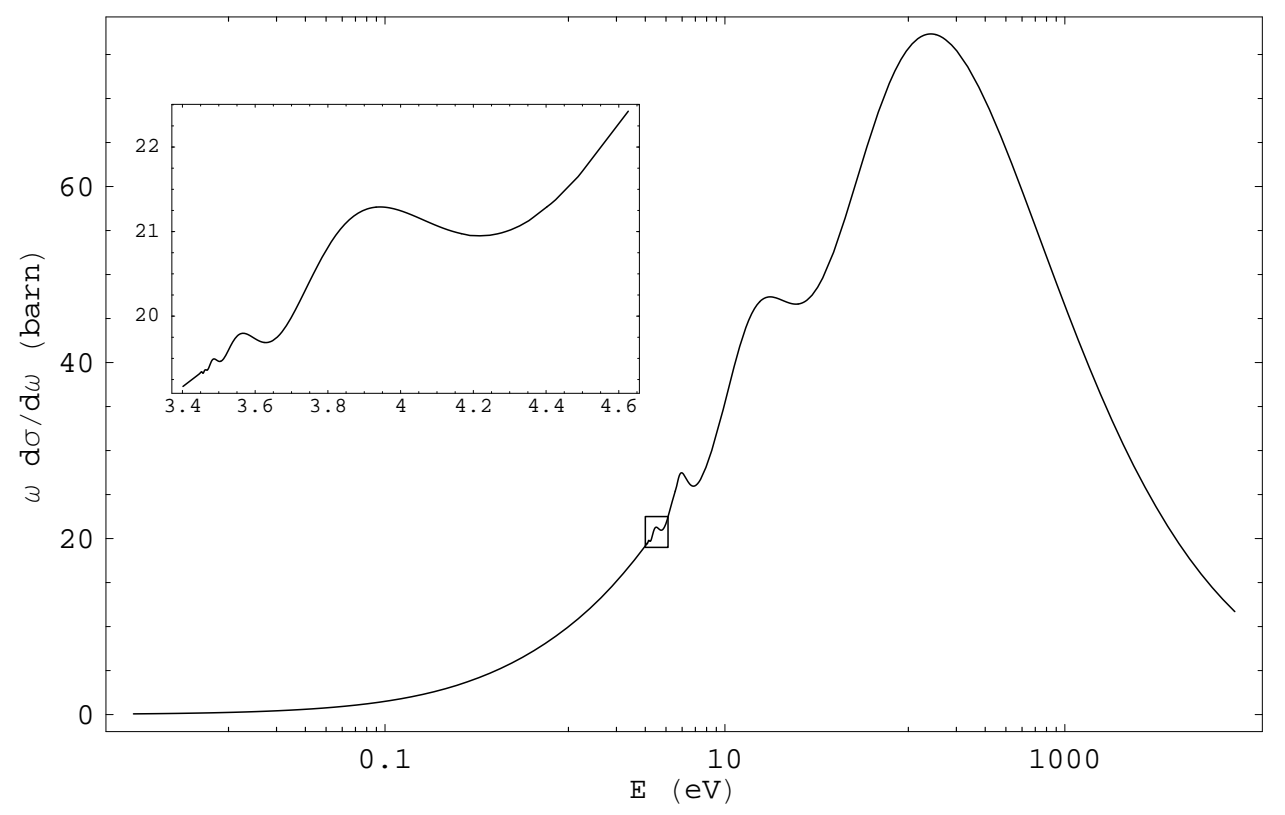

Figure 2. Energy dependence of the classical bremsstrahlung cross section in the soft-photon limit, for $\mathrm{Al}$ in a Hartree-Fock potential, showing detail (see insert) of the region near the energy $E$ where the maximum and the divergence of $\Phi(E, L)$ as a function of $L$ merge, as discussed in section 3.1. (Note, this is simply proportional to $\mathcal{I}_{1}^{\mathrm{s}-\mathrm{ph}}$ of figure 1.)

(15) is a monotonic, decreasing function of $L$, in the screened case the scattering angle shows various structures, depending on the incident electron energy and the properties of the particular atomic potential. As an example we plot in figure 3 the scattering angle, considered as a function of $L$, for a number of energies for Al. We have chosen $\mathrm{Al}$ because it illustrates quite clearly all the features of the scattering angle which we want to discuss in this section.

For high energies the scattering angle in screened potentials is a monotonic decreasing function of $L$, as in the Coulomb case. For lower energies (starting at $\sim 500 \mathrm{eV}$ for Al) $\Phi$ initially increases from its starting value of $\pi$ and a flat smooth maximum can be observed (see, e.g., $E=60 \mathrm{eV}$ in figure 3 ). $\ddagger$ As the energy decreases, the maximum occurs at larger $L$, reaching $L_{\max } \approx 2$ at $E=60 \mathrm{eV}$, and its height slightly increases. With further decrease of energy a peak develops above the smooth maximum (see, e.g., $E=26 \mathrm{eV}$ ), becoming in the $\mathrm{eV}$ range very pronounced (see the left maximum for $E=6$ and $4.9 \mathrm{eV}$ ), approaching (but in Al not reaching) a divergent behaviour, and its position shifts to the left (opposite to the motion of the smooth maximum). Simultaneously, another structure develops. It is first seen as bump on the right shoulder of the maximum (e.g., see at $E=6 \mathrm{eV}$ ). Below a certain energy $E_{\mathrm{M}}=4.9 \mathrm{eV}$ this structure is a divergence. The divergence occurs at a particular angular momenta $L_{\mathrm{t}}(E)\left(L_{\mathrm{t}}\left(E_{\mathrm{M}}\right) \equiv L_{\mathrm{M}}\right)$ determined by the energy $E$ (see further discussion below). In $\mathrm{Al}$ at $E=E_{\mathrm{M}}$ the divergence coexists with

$\ddagger$ A small slope of $\Phi$ at small $L$ results in enhancement of backward scattering; this phenomenon is called Coulomb glory [9, 10]. 


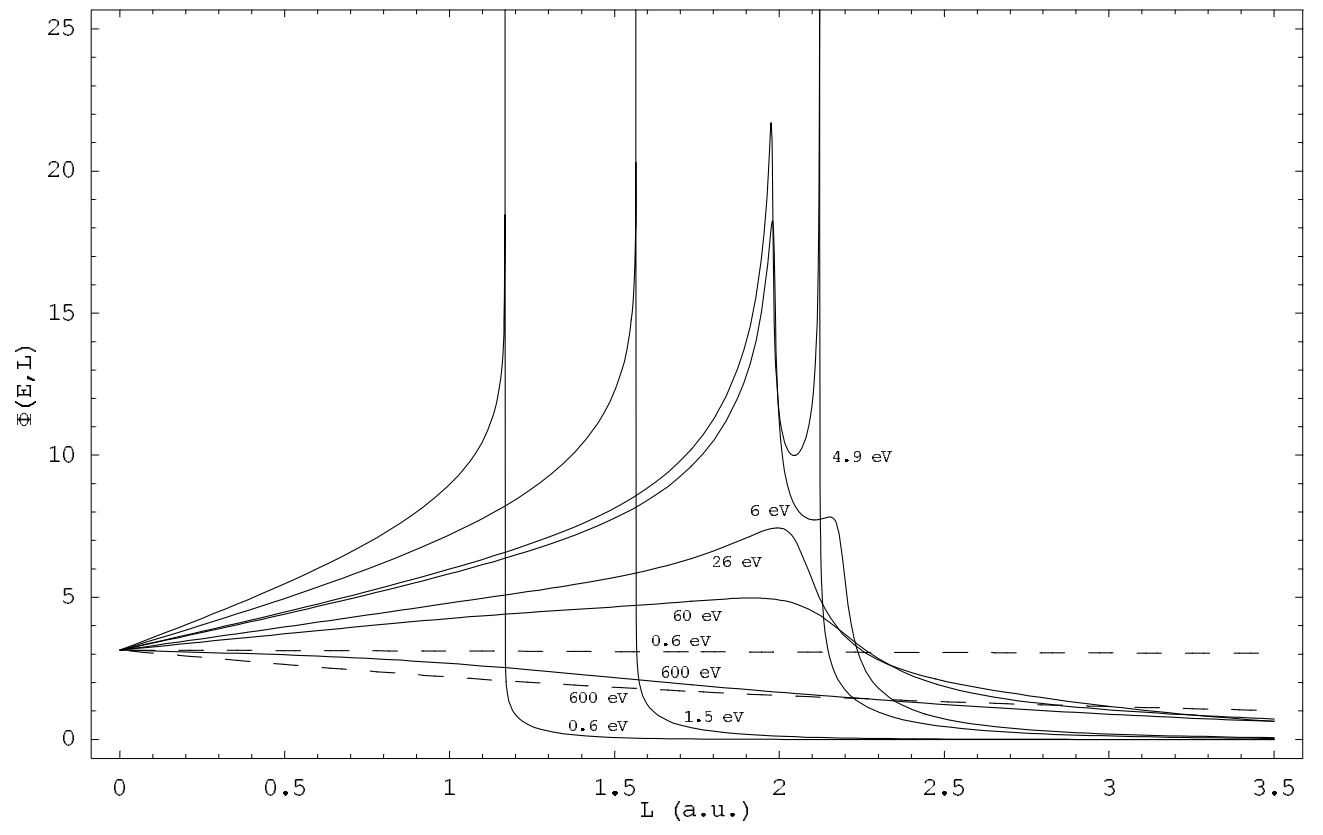

Figure 3. The scattering angle in $\mathrm{Al}$ as a function of $\mathrm{L}$ for Coulombic (dashed lines) and screened (solid lines) potentials, for a number of incident electron energies.

the semi-divergent maximum, which is located at $L<L_{\mathrm{M}}$. With further decrease of energy, $L_{\mathrm{t}}(E)$ decreases and the divergence moves to the left, eventually at certain energy coinciding with and "swallowing" the sharp maximum and becoming the only observable structure in the scattering angle (see, e.g., $E=1.5 \mathrm{eV}$ ). For $L>L_{\mathrm{t}}(E)$ the scattering angle has an abrupt decrease (fall off) toward 0.

We have found four general types of behaviour of the scattering angle in neutral atoms. In all four situations one divergence develops below a certain energy (trapping divergence, as discussed below). The first type of behaviour we found in $\mathrm{Na}$ and $\mathrm{Mg}$. In these atoms two divergences of the scattering angle can be observed in some (narrow) energy range. In the second type of behaviour $(\mathrm{Al}, \mathrm{Sr})$ the divergence and a very sharp (semi-divergent) maximum coexist, but the second divergence does not develop completely. (The left maximum in $\mathrm{Al}$ which is seen at $E=6 \mathrm{eV}$ and $E=4.9 \mathrm{eV}$ in figure 3 is approaching a divergent nature, as opposed to the smooth maximum at $E=60 \mathrm{eV}$, but it never becomes a divergence.) In the third type (Be, $\mathrm{Si}, \mathrm{P})$ the divergence coexists with the smooth maximum, which does not develop a divergent or near divergent nature. In a fourth group of atoms $(\mathrm{Ne}, \mathrm{Ca})$ the broad maximum never coexists with the divergence. We will not attempt to discuss the details of these behaviours here. However, we give some further discussion of the nature of the divergences and semi-divergences of the scattering angle and their connection to the features of the effective potential in subsubsection 3.1.3. Let us note that the behaviour of the scattering angle in ions would be different because of uncompensated nuclear charge and the existence of a long range Coulomb tail, as well as because of the different characteristic sizes of ions (ions are generally smaller). In this paper we have restricted ourselves to neutral atoms. 
Examining the general behaviour of the scattering angle with $L$ as one varies $E$, one can identify four main features: rise, (smooth) maximum, divergence, and (abrupt) decrease. Let us now briefly discuss these characteristics one by one.

3.1.1. The rise In the pure Coulomb potential and in a screened potential with Coulombic asymptotics at the origin, for any $E>0$ the scattering angle has the limit, for vanishing angular momentum, $\Phi \rightarrow \pi[9]$. However, for $E \rightarrow 0$, in the Coulomb case $\Phi \rightarrow \pi$ for all finite $L$, as opposed to the screened case where $\Phi \rightarrow 0$ for $E \rightarrow 0$ and $L \neq 0$. This difference is due to the fact that the Coulombic potential is long range; the effective potential (10) approaches zero from below at large $r$ for all $L$, so that even for $E \rightarrow 0$ the particle will be attracted and scattered back. In the screened case the effective potential approaches zero from above for large $r$ for all $L \neq 0$, so that when $E \rightarrow 0$ one does not overcome the centrifugal barrier: the particle can not feel the potential.

The deviation of $\Phi$ from $\pi$ for small $L$ can be expanded in power series:

$$
\Phi(E, L)=\pi+C_{1} L+C_{2} L^{3}+\ldots
$$

The expansion coefficients depend on energy and shape of the potential. In a screened potential at small energies $C_{1}>0$ and $\Phi$ increases linearly with $L$. One can find an expression for $C_{1}$ by exploiting the Coulombic behaviour of the potential at the origin: for any given accuracy one can find a distance $r_{\mathrm{c}}$ until which the screened and Coulombic potentials can be considered equal. Then for all $L<L_{\mathrm{c}}=\sqrt{2 m Z e^{2} r_{\mathrm{c}}}$ the turning point of the motion can be considered Coulombic, $r_{0}(E, L)=r_{0}^{\operatorname{Coul}}(E, L)$, within a certain accuracy. Consequently, the integration interval can be divided into two parts: (i) $\left[r_{0}^{\operatorname{Coul}}(E, L), r_{\mathrm{c}}\right]$ (where the atomic potential is considered Coulombic) and (ii) $\left(r_{c}, \infty\right)$ (where the atomic potential is screened). Then

$$
C_{1}=2 \lambda\left[\zeta \int_{r_{\mathrm{c}}}^{\infty} \frac{\mathrm{d} r}{r^{2} \sqrt{1-\frac{V(r)}{E}}}-\sqrt{1+2 \frac{\zeta}{r_{\mathrm{c}}}}\right] \text { for } L \ll \lambda^{-1} \frac{r_{\mathrm{c}}}{Z} .
$$

In fact, this linear approximation for $\Phi$ works well even at larger $L$ in the screened case.

In the pure Coulomb case $C_{1}=-2 \lambda$ and $\Phi^{\text {Coul }} \leq \pi$ :

$$
\Phi^{\operatorname{Coul}}(E, L) \simeq \pi-2 \lambda L \quad \text { for } \quad L \rightarrow 0,
$$

However, for screened potentials, since $\left.|V(r)|<\left|V^{\operatorname{Coul}}(r)\right|\right)$, the positive first term in (18) can be bigger than the negative second term, leading to one of the most characteristic screening features: $\Phi$, always smaller than or equal to $\pi$ in the Coulomb case, can reach values larger than $\pi$ in the screened case. A physical explanation of this fact is as follows.

For small distances the screened potential can be described as the Coulombic potential plus a constant energy shift $\delta E$ [11]. Thus, at small distances the particle moves in the Coulomb potential with an effective energy $E_{\text {eff }}=E-\delta E$. Roughly, the trajectory can be described (at least at small $L$ ) as a piecewise function: inward motion along a straight line, motion along a Coulomb trajectory, and outward motion along a straight line. Although the energy shift $\delta E$ is small in comparison to the magnitude of the potential $Z / r$, it can be larger than the physical energy $E$ of the particle. In this case the Coulomb trajectory in the interior region would be an ellipse 
with the major semi-axis $a \equiv \zeta^{-1}=Z /\left(2\left|E_{\text {eff }}\right|\right)$, rather than a hyperbola. If, further, $a$ is small enough then half or more of the ellipse lies within the region of validity of the effective-energy Coulomb approximation, giving a trajectory rotation angle greater than $2 \pi$ and, consequently, the scattering angle $\Phi>\pi$. In fact there is also a transitional region of deviation from elliptical behaviour which contributes to the scattering angle. These behaviours are illustrated in figure 4.

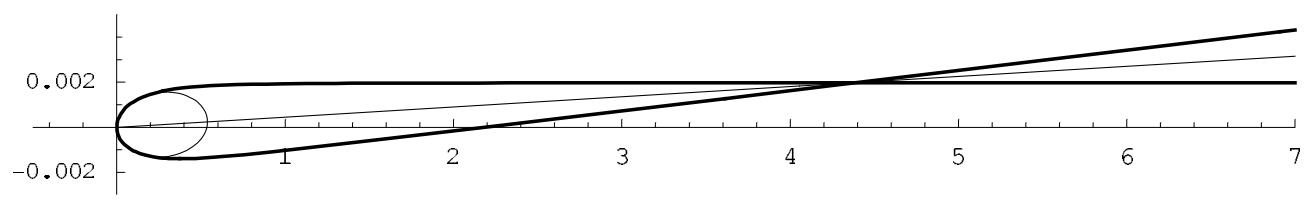

Figure 4. The (numerical) trajectory for an electron of $E=350 \mathrm{eV}$ (thick line) in a Hartree-Fock potential of $\mathrm{Al}$ and an elliptic (Coulomb) trajectory for $E_{\text {eff }}=-650 \mathrm{eV}$ (thin line) $(\delta E=1 \mathrm{keV})$; in both cases $L=0.01$ a.u. The straight solid line is the symmetry axis of the trajectory; the coordinate axes are marked in atomic units. Note that the y-scale in the figure is greatly expanded; in the actual scattering plane the ellipse is very prolate.

3.1.2. The smooth maximum The scattering angle, considered as an $L$-dependent function, must have a maximum whenever the initial slope is positive, since $\Phi(E, L=$ $0)=\pi$ which is greater than $\Phi(E, L \rightarrow \infty)=0$. We do not have a good physical parametrization of the behaviour. Our numerical results suggest that the maximum becomes higher and its position moves towards larger $L$ as the energy decreases. The trapping divergence (and in some cases other structures) starts to develop in the $\mathrm{eV}$ range of energies. These two features behave independently and can be distinguished by the opposite directions of motion of their position with energy. The smooth maximum can not occur to the right of the trapping divergence, where the rapid fall-off of the scattering angle occurs.

3.1.3. The trapping divergence Below a certain energy, characteristic of a given specified potential, there is a divergence in the scattering angle $\Phi$ which is connected to the orbiting (trapping) phenomenon. To understand this, we need to discuss some features of realistic screened neutral atom potentials. The effective potential (see figure 5) in the screened case can be partitioned into two manifolds, depending on $L$ : (i) $L>L_{\mathrm{M}}$, where $V_{\text {eff }}(r, L)$ is monotonically decreasing for all $r$, and (ii) $L<L_{\mathrm{M}}$, where $V_{\text {eff }}(r, L)$ has a barrier for each $L$. (Note that in the point Coulomb case the effective potential never has a barrier.) These two manifolds are separated by a curve $V_{\text {eff }}\left(r, L_{\mathrm{M}}\right)$ with an inflexion point at $r=r_{\mathrm{M}}$. This defines $L_{\mathrm{M}}$ and $r_{\mathrm{M}}$. We call $E_{\mathrm{M}}=V_{\mathrm{eff}}\left(r_{\mathrm{M}}, L_{\mathrm{M}}\right)$ the maximum trapping energy. In the $L<L_{\mathrm{M}}$ manifold, for any fixed energy $E<E_{\mathrm{M}}$ there will be an angular momentum $L_{\mathrm{t}}(E)$ $\left(\lim _{E \rightarrow E_{\mathrm{M}}} L_{\mathrm{t}}(E)=L_{\mathrm{M}}\right)$ for which the height of the barrier coincides with the energy $E$. Therefore, for any energy below (or near) $E_{\mathrm{M}}$, for angular momenta in the vicinity of $L_{\mathrm{t}}(E)$ (or $L_{\mathrm{M}}$ ) the electron will make several revolutions around the scattering center before escaping the potential. This phenomenon is known as trapping (or orbiting). At $L=L_{\mathrm{t}}(E)$ the particle is captured (makes an infinite number of rotations) and the scattering angle is infinite.

This is a typical picture. We should note, however, that the effective potentials $V_{\text {eff }}$ of some atoms may show more complicated behaviour. In some (narrow) angular 


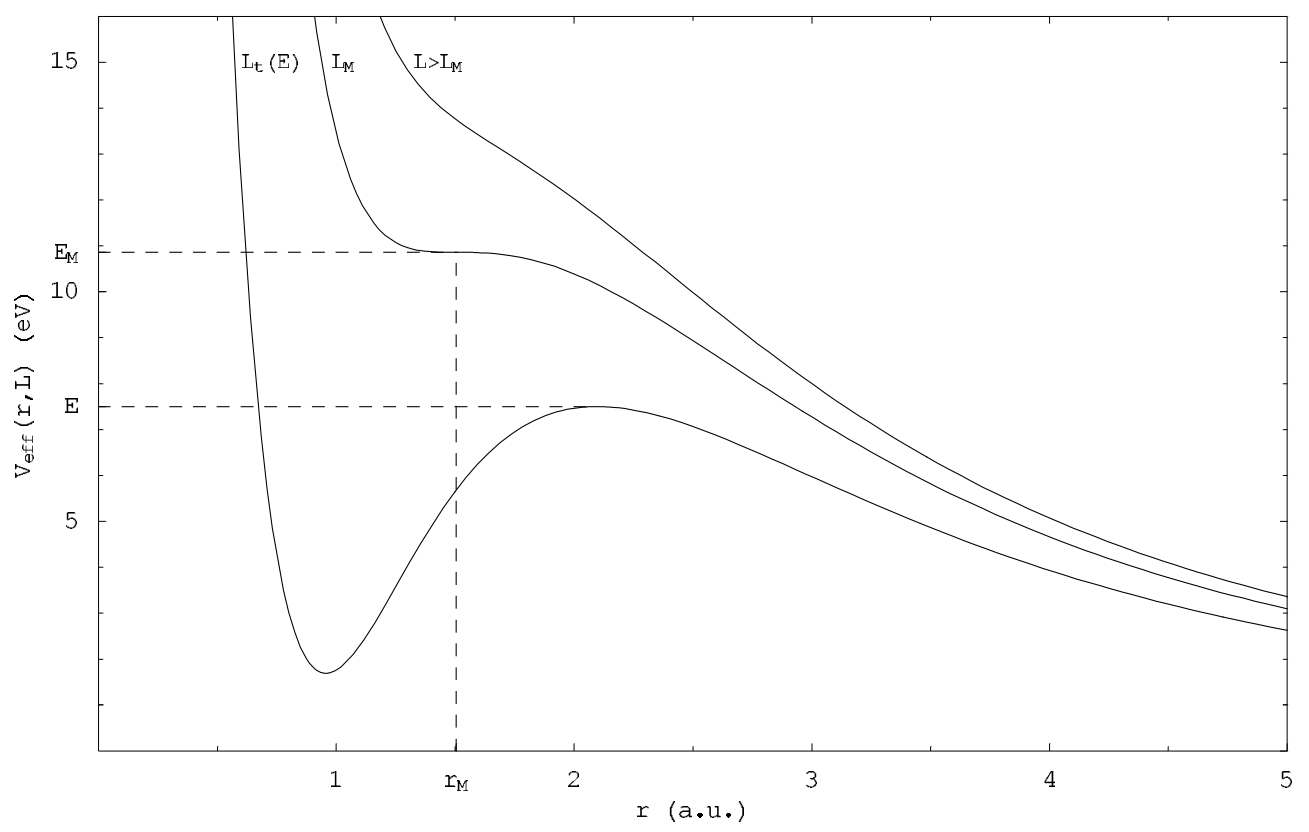

Figure 5. A typical effective potential (10) for a neutral atom (example of $\mathrm{P})$. For any energy $E<E_{\mathrm{M}}$ there is an angular momentum $L_{\mathrm{t}}(E)<L_{\mathrm{M}}$ such that the height of the potential at the top of the barrier coincides with $E$. For $L=L_{\mathrm{t}}\left(E_{\mathrm{M}}\right) \equiv L_{\mathrm{M}}$ the effective potential has an inflexion point. For $L>L_{\mathrm{M}}$ the effective potential is a monotonic decreasing function of $r$. We call $E_{\mathrm{M}}$ the maximum trapping energy.

momentum range they can have two wells and, correspondingly, two barriers, as shown in figure 6. In such atoms the scattering angle $\Phi$ considered as a function of $L$ at fixed energy $E$ can manifest two divergences. This is because trapping at energy $E$ can occur for two different values of angular momentum, for one at which the height of the inner barrier is $E$, and for the other the height of the outer barrier is $E$ (see inset in figure 6). This does not happen in $\mathrm{Al}$ because for any given $L$ the inner barrier is always lower than the outer one and trapping on the inner barrier can not take place, as the inner barrier is "screened" by the outer one. Instead, the scattering angle in $\mathrm{Al}$ shows one divergence and one semi-divergence. The inner barrier is only slightly lower than the outer one, and it can significantly influence the behaviour of the scattering angle, though not leading to a true divergence, as is seen in figure 3 for $E=6 \mathrm{eV}$ and $E=4.9 \mathrm{eV}$.

We have obtained two expressions (Appendix A) which describe the divergent or near-divergent behaviour of $\Phi(E, L)$. The first is valid for $E<E_{\mathrm{M}}$. It is similar to the expression given in [12] for the case $L<L_{\mathrm{t}}(E)$ and generalizes that formula for the case of any $L$. The second, describing the divergence occuring when $E \rightarrow E_{\mathrm{M}}$ $\left(E>E_{\mathrm{M}}\right)$ and $L \rightarrow L_{\mathrm{M}}$, was given in [6].

3.1.4. The fall off For energies which allow orbiting, the trapping divergence is followed by an abrupt decrease (fall off) of the scattering angle with increasing $L$. This can be explained in terms of the turning point behaviour. For a given $E<E_{\mathrm{M}}$ the effective potential has an outer barrier (see figure 5). If $L<L_{\mathrm{t}}(E)$ the height 

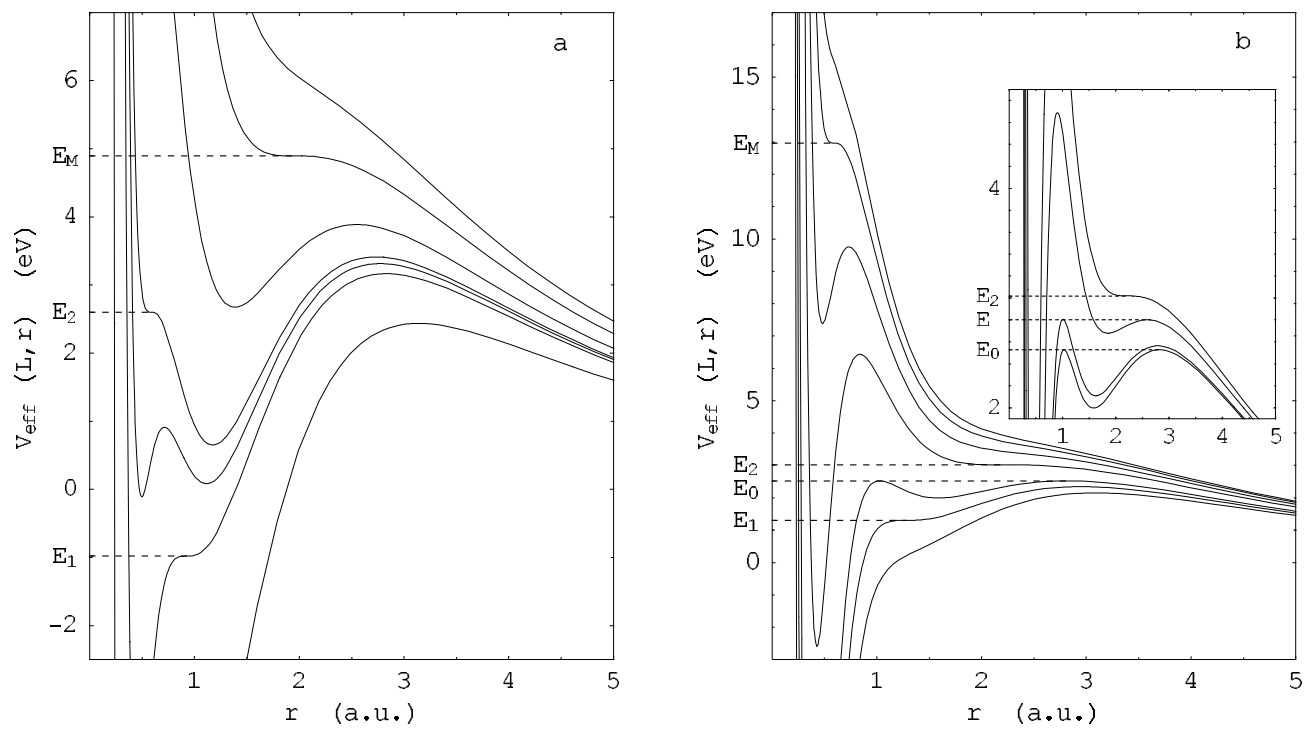

Figure 6. Double well structure in the effective potential (10) in (a) $\mathrm{Al}$ and (b) $\mathrm{Mg}$, for a number of angular momenta $(L=1.8,1.928,1.95,1.964,2.025,2.122$, 2.2 for $\mathrm{Al} ; L=1.73,1.768,1.798,1.865,1.905,1.933,1.95$ for $\mathrm{Mg}$ ). In accord with the definition (10) of the effective potential, the greater $L$ is, the higher $V_{\text {eff }}$. In contrast to the more typical case presented in figure 5 , here $V_{\text {eff }}$ has two barriers and two wells in some range of angular momentum. In such atoms there are three potential curves having an inflexion point, with corresponding energies denoted by $E_{1}, E_{2}$, and $E_{\mathrm{M}}$ (the maximal one). In $\mathrm{Al}$ for any given $L$ the inner barrier is always lower than the outer one; in $\mathrm{Mg}$ either the inner or the outer barrier can be higher, and at one angular momentum they are of equal height $E_{0}$. We note, that in $\mathrm{Mg}$ for any energy $E \in\left(E_{0}, E_{2}\right)$ there is one angular momentum at which the height of the inner barrier is equal to $E$ (and greater than the outer barrier), and another angular momentum at which the height of the outer barrier is equal to $E$, as shown in the inset of (b).

of the barrier is less than the energy $E$ and the electron passes over the barrier into the inner region of the potential. For $L>L_{\mathrm{t}}(E)$ the height of the barrier becomes larger than the energy $E$ and the turning point abruptly jumps from the inner region of the potential to the right side of the outer barrier, as shown in figure 7 . This leads to an abrupt change in electron trajectory: for $L<L_{\mathrm{t}}(E)$ the electron feels the attractive atomic potential and the scattering angle is large, while for $L>L_{\mathrm{t}}(E)$ the atomic potential can be considered small and the scattering angle is also small. The abruptness of change of the turning point decreases with increasing electron energy, and for $E>E_{\mathrm{M}} r_{0}(E, L)$ is a smooth function of $L$. This suggests that the fall off is less abrupt for larger electron energies. Above $E_{\mathrm{M}}$ the fall-off approaches the slower Coulombic fall-off behaviour.

Let us note in conclusion that even though the four features described above are characteristic of screened neutral potentials which are Coulombic when $r \rightarrow 0$ and which tend to zero faster than $1 / r^{2}$ for large $r$, their importance may vary from one element to another. The details of the features are also sensitive to the approximation used for the atomic potential (we have used Kohn-Sham and HartreeFock potentials, with and without polarizational tail). Since we have found these 


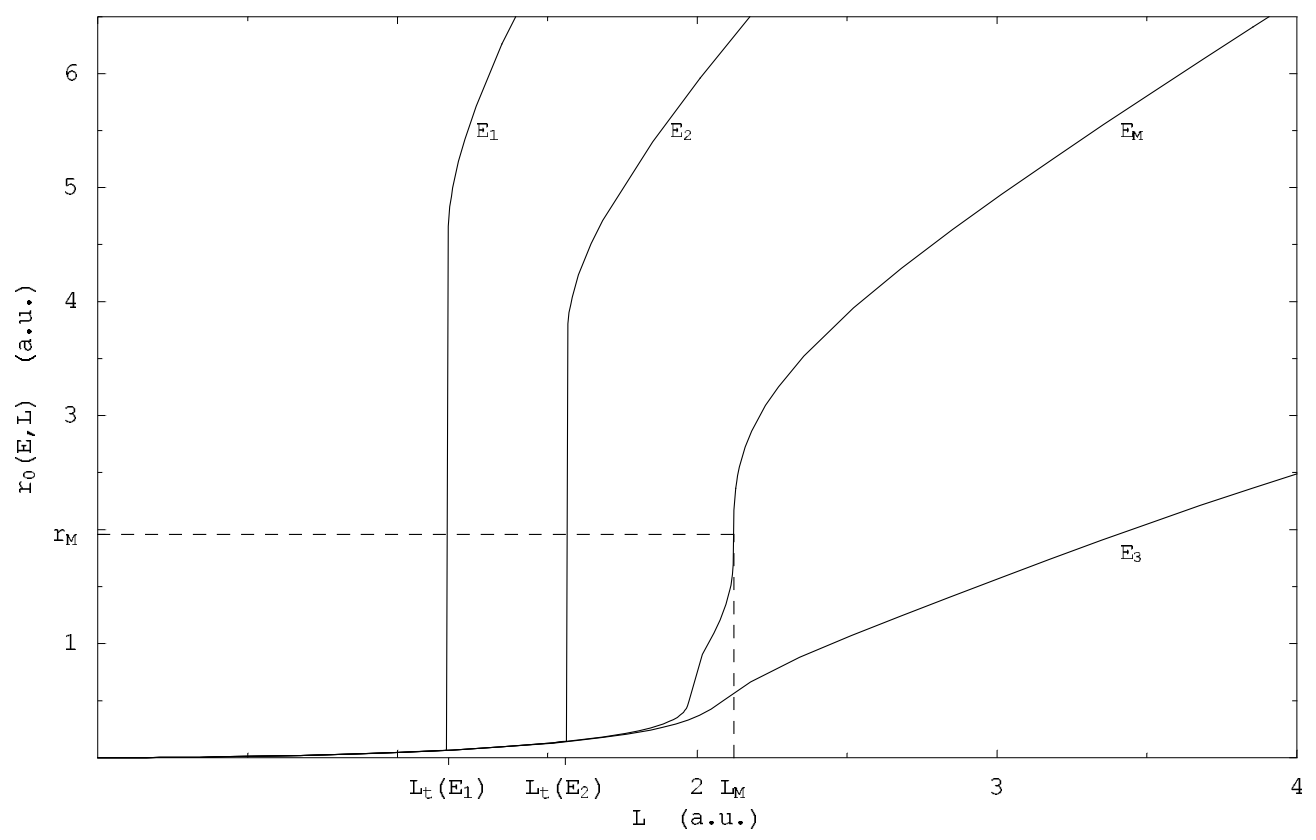

Figure 7. The turning point of a radial motion $r_{0}(E, L)$ in $\mathrm{Al}$ as a function of $L$ for several fixed energies: $E_{1}=0.6 \mathrm{eV}, E_{2}=1.5 \mathrm{eV}, E_{\mathrm{M}}=4.9 \mathrm{eV}$ (the maximal trapping energy), and $E_{3}=30 \mathrm{eV}$. The turning point abruptly increases at a certain $L=L_{\mathrm{t}}(E)$ for which trapping occurs. For $E>E_{\mathrm{M}}$ there is no trapping and the turning point is a smooth function of $L$.

features in polarization potentials, it is suggestive that our discussion remains relevant even when electron-electron correlations are included, as they should be in a realistic discussion at these low energies.

\subsection{The differential cross section and the asymmetry parameter}

Let us consider the impact of the features of the scattering angle discussed above on the bremsstrahlung cross section and asymmetry parameter. We shall first discuss the energy dependence of the spectrum $\omega \mathrm{d} \sigma / \mathrm{d} \omega$ and then that of the asymmetry parameter $a_{2}$.

Due to the long range of the potential in the Coulomb case the cross section in the soft photon limit is infinite for any electron energy. In contrast, in a screened neutral (short-range) atomic potential the cross section vanishes for small and large energies. A simple physical explanation of these two limits can be given as follows. For large energies an electron is moving too fast to be scattered and to emit a significant amount of radiation. For small energies, due to the centrifugal barrier in a screened potential, an electron stays at infinite distance from the atom for all $L$ (providing that the attractive atomic potential goes to zero faster than $1 / r^{2}$, i.e. the effective potential at infinity is repulsive). The exception is the case $L=0$, where there is no centrifugal barrier, and the limiting value of the scattering angle $\Phi(E, L)$ is $\pi$. However this single finite non-zero value of $\Phi$ can not lead to a finite cross section. Mathematically, the limiting cases can be obtained from equations (2), (12) and the corresponding behaviour of $\Phi(E, L)$. 
Between these two limits, with increasing energy we observe a rise to a peak, modulated by several oscillations. The amplitude of the oscillations increases with energy. The number of oscillations is different in different atoms. In $\mathrm{Al}$ we could see up to seven oscillations with our present level of accuracy.

The origin of these oscillations is in the lack of contributions to the integral (12) from some ranges of angular momentum, at certain electron energies. Such a phenomenon has a quantum mechanical analogue. In the quantum description, the electron angular momenta are quantized and the bremsstrahlung cross section is expressed in terms of a sum (rather than an integral) over $L$. At particular energies particular terms in the sum can vanish due to zeroes in the radiation matrix elements. This leads to minima in the energy dependence of the quantum bremsstrahlung cross section [6].

In the classical case, the integrand from (12),

$$
J_{1}(E, L) \equiv L[1-\cos \Phi(E, L)]
$$

cancels every time $\Phi(E, L)$ as a function of $L$ crosses $2 n \pi$. Such zeroes at particular $L$ in (20), which will occur over broad ranges in $E$, are not directly connected to the minima in the cross section. However, if $\Phi(E, L) \simeq 2 n \pi$ in a wider range, this can lead to a minimum in the cross section. This can happen, for instance, when $\Phi(E, L)$ has a broad maximum (minimum) near $2 n \pi$, see, e.g., $E=26 \mathrm{eV}$ in figure 3. Another possibility can be a "shoulder" on a wing of the maximum, as occurs in $\mathrm{Al}$ for $E \approx 6.5 \mathrm{eV}$ (figure 3). The former situation gives rise to the first minimum in the cross section for $\mathrm{Al}$ atom, while the latter situation gives the second minimum of the cross section (moving from larger to smaller energies). Analogously, a maximum of the cross section occurs when the scattering angle is approximately $(2 n+1) \pi$ in a wider region of $L$. Such situations appear as a result of combination of broad smooth maximum and the divergence in different stages of its development. We emphasize that the trapping divergence (or quasi-divergence) itself, as well as the rise and fall-off features, are not responsible for the structures, due to the rapid oscillations of $J_{1}(E, L)$ in the vicinity of the divergence [6].

In figures 8 and 9 we show how (20) and (as inset figures) the corresponding scattering angle $\Phi(E, L)$ behave as functions of $L$ at two energies, one corresponding to a maximum and one to a minimum in the cross section. The contributions to (12) from different $L$ regions are given in the inset histograms. The regions $I, I I$, and III can be defined somewhat arbitrarily; we use them only to draw attention to the relevant interval of $L$ which determines the behaviour of the cross section. Namely, it can be seen from the figures that the lack of contribution from region $I I$ determines the minimum in the cross section. We should note, however, that the minimum occurs in spite of the opposite contributions from regions $I$ and $I I I$.

We do not see structures in the cross section if there is only a smooth maximum or an isolated divergence in $\Phi$, but rather when there is a combination of these features. The structures only appear in a certain energy interval, which ranges from few electron-Volts up to few hundreds of electron-Volts depending on the atom. In $\mathrm{Al}$ the amplitudes and periods of the oscillations associated with these structures generally increase with energy, but in other atoms the behaviour can be less regular.

In figure 1 we show the asymmetry parameter $a_{2}^{\mathrm{s}-\mathrm{ph}}$ which is the ratio of $\mathcal{I}_{2}^{\mathrm{s}-\mathrm{ph}}$ and $\mathcal{I}_{1}^{\mathrm{s}-\mathrm{ph}}$, also plotted in the same figure. Both $\mathcal{I}_{2}^{\mathrm{s}-\mathrm{ph}}$ and $\mathcal{I}_{1}^{\mathrm{s}-\mathrm{ph}}$ have structures in their energy dependence. We have already discussed the origin of these structures in $\mathcal{I}_{1}^{\mathrm{s}-\mathrm{ph}}$, which is proportional to the cross section. The structures in $\mathcal{I}_{2}^{\mathrm{s}-\mathrm{ph}}$ have 


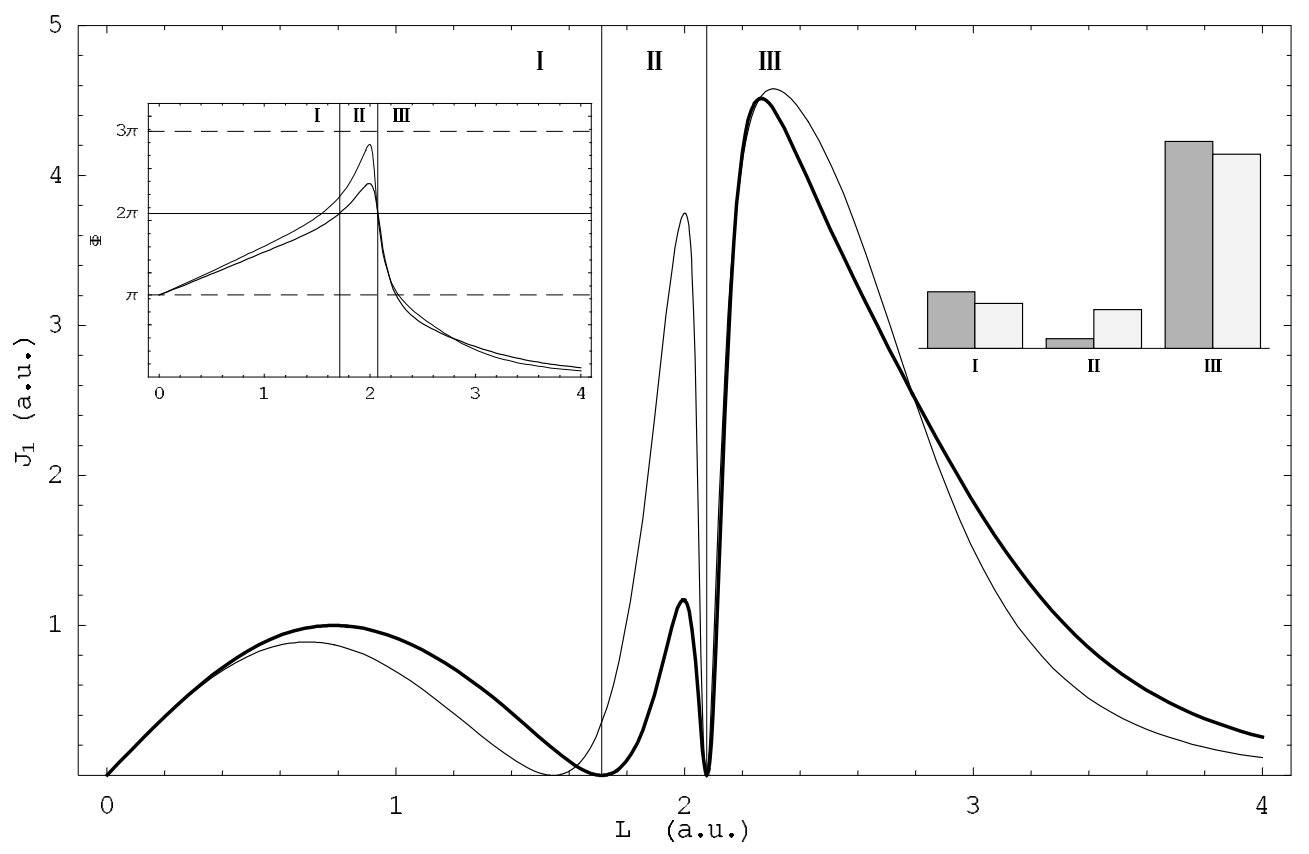

Figure 8. The subintegral expression $J_{1}(20)$ for $\mathrm{Al}$ as a function of $L$ for two energies, corresponding to the first minimum (thick line, $E=26 \mathrm{eV}$ ) and maximum (thin line, $E=19 \mathrm{eV}$ ) of the cross section. In the inset we give the scattering angle $\Phi(E, L)$ as a function of $L$ for these two energies (again, thick line corresponds to the minimum, thin line to the maximum). We introduce three intervals in $L: \mathrm{I}-L \in\left(0, L_{\mathrm{I}}\right), \mathrm{II}-L \in\left(L_{\mathrm{I}}, L_{\mathrm{II}}\right)$, and III $-L \in\left(L_{\mathrm{II}}, \infty\right) . L_{\mathrm{I}}$ and $L_{\text {II }}$ are the solutions of $\Phi(E, L)=2 \pi$ for $E=26 \mathrm{eV}$. The result of integration over these three regions is given in the histogram (darker bars correspond to the minimum, $E=26 \mathrm{eV}$ ).

the same origin and are located at approximately the same energies, but the minima are deeper due to the additional factor $(3 \cos \Phi-1)$. Thus, $a_{2}^{\text {s-ph }}$ has minima in absolute value located at the same energies as the minima in $\mathcal{I}_{2}^{\mathrm{s}-\mathrm{ph}}$. We note that the asymmetry parameter has additional small structures coming from the somewhat shifted energies at which $\mathcal{I}_{1}^{\mathrm{s}-\mathrm{ph}}$ and $\mathcal{I}_{2}^{\mathrm{s}-\mathrm{ph}}$ have minima.

The limiting values of the asymmetry parameter in the soft photon case at small and large incident electron energies,

$$
\lim _{E \rightarrow 0} a_{2}=\lim _{E \rightarrow \infty} a_{2}=1,
$$

can be obtained from the equations (5), (12), (13), and the corresponding behaviour of $\Phi(E, L)$. These limiting values are reached far beyond the energy range where the structures occur, and they are not exhibited in figure 1.

\section{Conclusions}

We have investigated the origin of the structures in the differential cross section and in the asymmetry parameter in the classical bremstrahlung process, using the soft-photon limit case $\omega \rightarrow 0$. We show that the features of the scattering angle associated with the classical trapping phenomenon do not contribute to the structures. We find that in the 


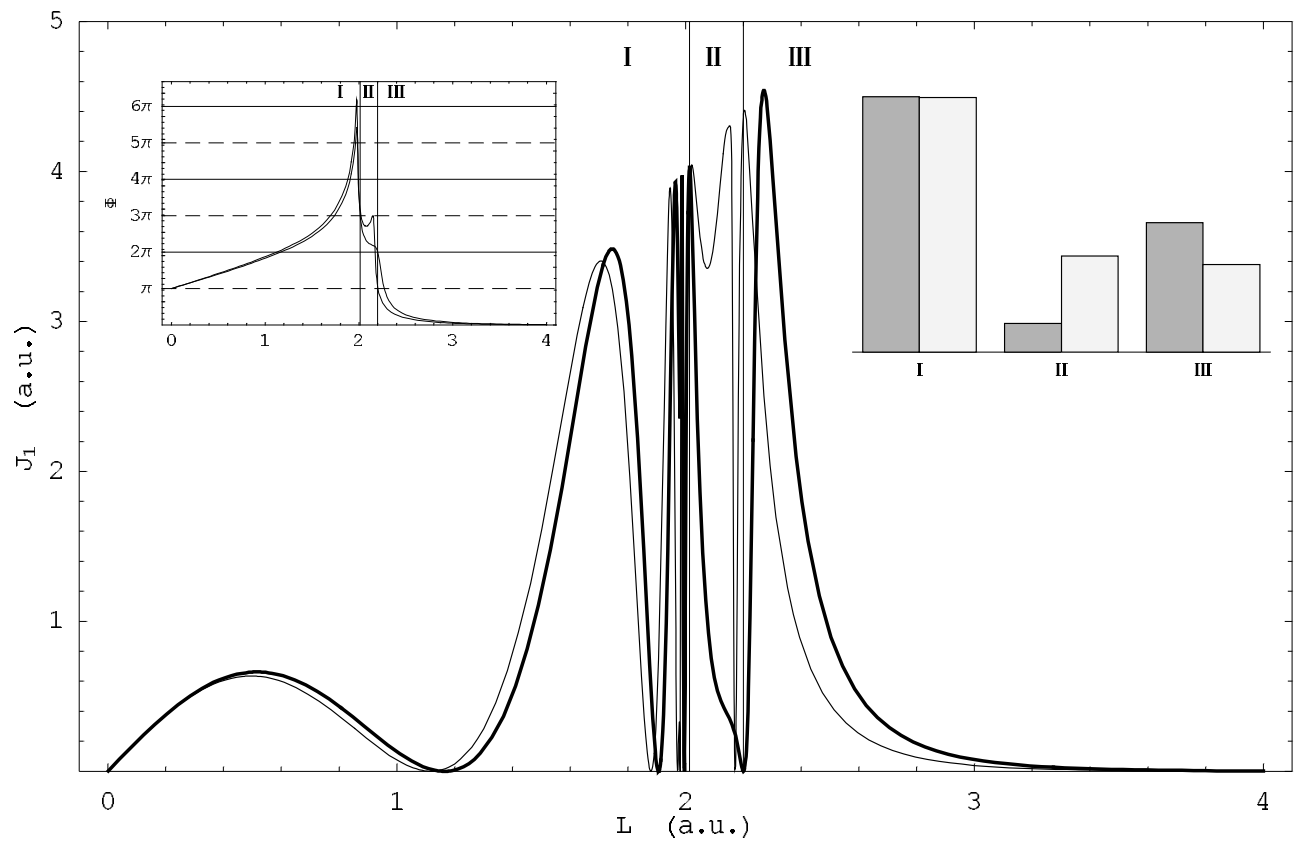

Figure 9. Same as in the figure 8 , but for the energies corresponding to the second minimum (thick line, $E=6.5 \mathrm{eV}$ ) and maximum (thin line, $E=5.5 \mathrm{eV}$ ) of the cross section. $L_{\mathrm{I}}$ and $L_{\mathrm{II}}$ are defined here by the following equations: $\Phi\left(E, L_{\mathrm{I}}\right)=3 \pi$ and $\Phi\left(E, L_{\mathrm{II}}\right)=2 \pi$ for $E=6.5 \mathrm{eV}$.

classical case, as in the quantum case, we can understand the oscillations with energy of $\omega \mathrm{d} \sigma / \mathrm{d} \omega$ and $a_{2}$ in terms of the small contribution of certain angular momenta at certain energies (in the quantum case these correspond to the energies at which some dominant radial matrix elements have zeroes). We have also examined how these structures can be understood classically in terms of features of elastic scattering, and we have analyzed the relative importance of the different features. The quantum and classical results are evidently related, but the precise relationship between quantum and classical bremsstrahlung in the soft-photon limit remains to be established.

\section{Acknowledgments}

Two of the authors (AF and OO) would like to thank the University of Pittsburgh for hospitality. We are grateful to Dr. A V Korol and to Dr. C D Shaffer for fruitful discussions and to Dr. J P J Carney for constant help and advice in computer issues.

\section{Appendix A.}

We give two expressions which describe the divergent or near-divergent behaviour of $\Phi(E, L)$. We shall call $\Phi^{\operatorname{div}}(E, L)$ the divergent (or near divergent) part of $\Phi(E, L)$ for $L$ near $L_{\mathrm{t}}(E)$.

For $E<E_{\mathrm{M}}$ the effective potential has a barrier (see figure 5) and when $L \rightarrow L_{\mathrm{t}}(E)$ the main contribution to the scattering angle comes from the $\Delta r$ - vicinity 
of the maximum, where the potential can be approximated by a parabola. Then, generalizing and completing [12],

$$
\begin{aligned}
\Phi^{\operatorname{div}}(E, L)= & \frac{1}{1+\Theta\left(L-L_{\mathrm{t}}(E)\right)} \sqrt{\frac{2}{\mu \gamma_{2}\left(L_{\mathrm{t}}(E)\right)}} \frac{L_{\mathrm{t}}(E)}{r_{\mathrm{t}}^{2}(E)} \times \\
& \ln \frac{4 m \gamma_{2}\left(L_{\mathrm{t}}(E)\right) r_{\mathrm{t}}^{2}(E) \cdot(\Delta r)^{2}}{L_{\mathrm{t}}(E)\left|L-L_{\mathrm{t}}(E)\right|},
\end{aligned}
$$

where $\Theta\left(L-L_{\mathrm{t}}(E)\right)$ is the Heaviside function of argument $\left(L-L_{\mathrm{t}}(E)\right)$ and

$$
\gamma_{2}\left(L_{\mathrm{t}}(E)\right)=-\frac{1}{2} \frac{\partial^{2} V_{\mathrm{eff}}}{\partial r^{2}}\left(r_{\mathrm{t}}(E), L_{\mathrm{t}}(E)\right) .
$$

For $E \rightarrow E_{\mathrm{M}}\left(E>E_{\mathrm{M}}\right)$ the effective potential has an inflexion point when $L \rightarrow L_{\mathrm{M}}$ and $[6]$

$$
\begin{gathered}
\Phi^{\operatorname{div}}(E, L)=\sqrt{\frac{2}{m}} L \\
\left\{\frac{\gamma_{3}\left(L_{\mathrm{M}}\right)}{2}\left[\frac{6}{\gamma_{3}\left(L_{\mathrm{M}}\right)}\left(E-E_{\mathrm{M}}-\frac{L^{2}-L_{\mathrm{M}}^{2}}{2 m r_{\mathrm{M}}^{2}}\right)\right]^{2 / 3}+\frac{L^{2}-L_{\mathrm{M}}^{2}}{m r_{\mathrm{M}}^{3}}\right\}^{-1 / 2} \\
\left(\frac{\sqrt{\Delta r}}{r_{\mathrm{M}}\left(r_{\mathrm{M}}+\Delta r\right)}+\frac{1}{r_{\mathrm{M}}^{3 / 2}} \arctan \sqrt{\frac{\Delta r}{r_{\mathrm{M}}}}\right),
\end{gathered}
$$

where

$$
\gamma_{3}\left(L_{\mathrm{M}}\right)=-\frac{\partial^{3} V_{\mathrm{eff}}}{\partial r^{3}}\left(r_{\mathrm{M}}, L_{\mathrm{M}}\right)
$$

Both analytical formulae fit well the numerical evaluation of the divergent part of the scattering angle.

\section{References}

[1] Kim L and Pratt R H 1987 Phys. Rev. A 3645

[2] Shaffer C D 1996 PhD thesis, (Pittsburgh: University of Pittsburgh)

[3] Jackson J D 1975 Classical Electrodynamics, $2^{\text {nd }}$ ed. (NY: John Wiley and Sons)

[4] Landau L D and Lifshitz E M 1959 The Classical Theory of Fields (Reading, Massachusetts: Addison-Wesley)

[5] Korol A V, Lyalin A G and Solovyov A V 1995 J. Phys. B: At. Mol. Phys. 284947

[6] Florescu A, Obolensky O I, Shaffer C D and Pratt R H 2001 in Application of Accelerators in Research and Industry, eds. J L Duggan and I L Morgan, p. 60,Sixteenth International Conference, Denton, Texas 2000 (Melville, New York: AIP Conference Proceedings 576)

[7] Kogan V I, Kukushkin A B, and Lisitsa V S, Phys. Rep. 213, 1 (1992)

[8] Florescu V, Steiner V, and Burlacu L, J. Phys. B: At. Mol. Phys. 20, 427 (1987)

[9] Demkov Yu N, Ostrovsky V N and Telnov D A 1984 Zh. Eksp. Teor. Fiz. 84442 (Engl. transl. 1984 Sov. Phys. - JETP 59 257)

[10] Demkov Yu N and Ostrovsky V N 2001 J. Phys. B: At. Mol. Opt. Phys. 34 L595

[11] McEnnan J, Kissel L, and Pratt R H 1976 Phys. Rev. A 13532

[12] Mott N F and Massey H S W 1965 The theory of atomic collisions, Chapter 5 (Oxford: Clarendon Press) 